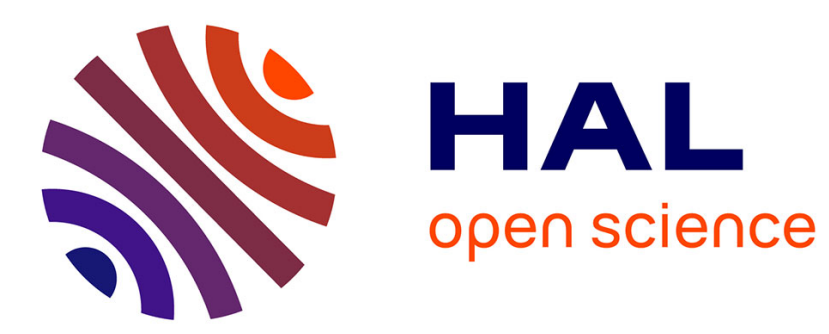

\title{
The role of eosinophils and basophils in allergic diseases considering genetic findings.
}

\author{
Rachel Nadif, Farid Zerimech, Emmanuelle Bouzigon, Regis Matran
}

\section{To cite this version:}

Rachel Nadif, Farid Zerimech, Emmanuelle Bouzigon, Regis Matran. The role of eosinophils and basophils in allergic diseases considering genetic findings.. Current Opinion in Allergy and Clinical Immunology, 2013, 13 (5), pp.507-13. 10.1097/ACI.0b013e328364e9c0 . inserm-00880260

\section{HAL Id: inserm-00880260 https://www.hal.inserm.fr/inserm-00880260}

Submitted on 3 Oct 2014

HAL is a multi-disciplinary open access archive for the deposit and dissemination of scientific research documents, whether they are published or not. The documents may come from teaching and research institutions in France or abroad, or from public or private research centers.
L'archive ouverte pluridisciplinaire HAL, est destinée au dépôt et à la diffusion de documents scientifiques de niveau recherche, publiés ou non, émanant des établissements d'enseignement et de recherche français ou étrangers, des laboratoires publics ou privés. 


\section{The role of eosinophils and basophils in allergic diseases considering genetic}

\section{findings}

Rachel Nadif ${ }^{\mathrm{a}, \mathrm{b}}$, Farid Zerimech ${ }^{\mathrm{c}, \mathrm{d}}$, Emmanuelle Bouzigon ${ }^{\mathrm{e}, \mathrm{f}}$, Regis Matran ${ }^{\mathrm{c}, \mathrm{d}}$

\section{Affiliations:}

${ }^{a}$ Inserm, Centre for research in Epidemiology and Population Health (CESP), U1018, Respiratory and Environmental Epidemiology Team, F-94807, Villejuif, France

${ }^{b}$ Univ Paris-Sud, UMRS 1018, F-94807, Villejuif, France

${ }^{c}$ CHRU de Lille, F-59000, Lille, France

${ }^{d}$ Univ Lille Nord de France, EA4483, F-59000, Lille, France

${ }^{e}$ Univ Paris Diderot, Sorbonne Paris Cité, Institut Universitaire d'Hématologie, F-75007, Paris, France

${ }^{f}$ Inserm, UMR-946, F-75010, Paris, France

\section{Correspondence to}

Rachel Nadif, PhD, Inserm, Centre for research in Epidemiology and Population Health (CESP), U1018, Respiratory and Environmental Epidemiology Team, F-94807, Villejuif, France. Phone number: 33 (0) 1455951 89, Fax number: 33 (0) 145595169

E-mail: rachel.nadif@inserm.fr 


\section{Purpose of review}

Basophils and eosinophils respectively represent less than 1 and $5 \%$ of white blood cells respectively. Their role in asthma and allergic inflammation remains incompletely defined. The present review addresses recent advances regarding the role of these two cell populations in allergic inflammation and asthma regarding both biological and genetic point of view.

\section{Recent findings}

Regarding eosinophils, the role of interleukin(IL)-25, IL-33 and thymic stromal lymphoprotein (TSLP) have been evidenced, and activation states of eosinophil Betal and Beta2 integrins have been found to correlate with measurement of eosinophil recruitment and pulmonary function in asthma. New insights in the biology of basophils concern their role as regulators of Th2 cell response through IL-4 expression or the differentiation of monocytes to macrophages, and their population heterogeneity in human. The transcription factor PU.1 was reported to be involved in controlling transcription of specific genes both in eosinophils and basophils. Candidate genetic studies on eosinophils have explored genes involved in the intracellular calcium influx and apoptosis. At the genome-wide level, studies identified genetic variants belonging to $I L 1 R L 1, T S L P$ and $I L-33$, and four loci with pleiotropic effects on eosinophil and basophil counts (GATA2 (3q21), MHC (6p21), HBS1L-MYB (6q23), and $E R G(21 \mathrm{q} 22))$.

\section{Summary}

Recent findings from biological and genetic studies on eosinophils and basophils highlight the role of epithelial cell-derived cytokines such as TSLP and IL-33 in asthma and allergic diseases.

\section{Keywords}

basophil, eosinophil, allergy, asthma, TSLP, IL-33, IL-25, integrins 


\section{INTRODUCTION}

Blood granulocytes are divided in three subsets: basophils, eosinophils and neutrophils. Both basophils and eosinophils are involved in allergic inflammation, and circulate at relatively low levels in the blood, making-up $0.1-1 \%$ and $1-5 \%$ of white blood cells respectively.

The contribution of basophils and eosinophils to allergic inflammation remains incompletely defined. Although described by Paul Ehrlich in 1879 and conserved in all vertebrates, including fish, birds and reptiles, the in vivo role of basophils is always elusive and was poorly investigated, and therefore sometimes considered as possibly redundant "circulating mast cells" [1]. In recent years, basophils have been considered as primary effector cells in allergic disorders such as asthma, hay fever and anaphylaxis. In contrast, extensive studies on eosinophils have revealed their contribution to host defense, immunomodulation, tissue remodeling and fibrosis, and in immunoregulatory and anti-inflammatory processes. Activation of eosinophils in asthmatic patients causes inflammation, airway remodeling, mucus production and airway hyperreactivity. The recruitment of eosinophils is related to the release of cytokines, chemokines and others products such as IL-3, IL-4, IL-5, IL-13, CCL11, CCL24, CCL26, TNFa, integrins, and histamine, released from different cells involved in the disorders (see Figure 1) [2]. Basophils may be recruited to sites of inflammation especially during immune responses against allergens and contribute to hypersensitivity reactions. Basophils are also known as regulators of $\mathrm{Th} 2$ responses in allergic inflammation and can play a role of antigen-presenting cells (APCs).

Many publications have been published on both the role of cytokines and other mediators in all aspects of eosinophil development, activation and survival, and cellular features of eosinophils. In contrast, the literature on basophils is scarce, and this imbalance is reflected in this review which focuses on the new insights of the role of basophils and eosinophils in allergic inflammation according to both biological and genetic findings in the last 18 months. 
In the first part of the review we highlight the role of three new epithelial cell-derived cytokines in the activation of eosinophils and basophils, then we discuss recent advances in biology regarding attraction, adhesion, migration and proliferation of eosinophils, the role of basophils in Th2 cell responses, the heterogeneity between basophil populations, and the similarity between the regulation of eosinophils and basophils. Published data relevant to the research of the genetic of eosinophils and basophils is discussed in the third part of this review.

\section{NEW INSIGHTS IN THE BIOLOGY OF EOSINOPHILS}

Recent literature highlights the role of epithelial cell-derived cytokines in the activation of eosinophils and reported new findings on the attraction, adhesion, migration and proliferation of eosinophils.

\section{The role of the airway epithelial cells}

Airway epithelial cells represent the first line of defense against allergens and various particles. Activation of the cells is a key triggering event in the recognition of inhaled allergens that activates the network of dendritic cells (DCs) to promote Th-2 immunity. Epithelial-derived cytokines and chemokines also activate innate immune cells such as basophils, mast cells, eosinophils and neutrophils [3]. More recent studies indicate an immunologic function of airway epithelium. A triad of cytokines, including interleukin (IL)25, IL-33 and Thymic stromal lymphoprotein (TSLP) - a cytokine associated with the development of multiple allergic disorders - is released by airway epithelial cells in response to various environmental factors (see Figure 1) [4].

IL-25 is produced by activated Th2 cells, epithelial cells and mast cells. IL-25 provokes the production of Th2 type cytokines (including IL-5) from Th2 cells and subsequently amplify the recruitment and survival of eosinophils in allergic patients. High expression of both IL-25 
and the IL-25 receptor has been detected in a recent study of bronchial and skin biopsies from allergic patients [5].

IL-33 has the potential to induce Th2 cytokine-mediated allergic inflammation. IL-33 is implicated in asthma, allergic conjunctivitis and anaphylactic responses. It has been shown that IL-33 is an essential biomarker in the development of ragweed-induced allergic rhinitis in mice [6] because ragweed-immunized and ragweed-challenged IL-33 -/- mice showed a significant reduction in the frequency of sneezing, total and ragweed-specific response, and accumulation of eosinophils and basophils in the nasal mucosa [6]. In a mouse model of allergic asthma, pretreatment with anti IL-33 results in decreases of serum IgE, eosinophils in broncho-alveolar fluid (BALF), eosinophilic infiltration into the lung and decreased airway hyperresponsiveness [7].

TSLP, member of the IL-2 family, is mainly synthesized from epithelial cells but also from basophils and mast cells. Overexpression of TSLP in mice caused a weak innate response and was insufficient for development of airway inflammation. On the contrary, both overexpression of TSLP and antigen challenge provoke airway eosinophilia and airway remodeling in a mice model [8]. TSLP seems to play an important role in Th2-type immune responses and airway inflammation likely through mechanisms involving the interface between the innate and adaptive immunity and/or adaptive immunity itself [8]. Some recent publications concerning the role of TSLP are mainly focused on atopic dermatitis, and infection diseases.

\section{Attraction of eosinophils in the airways and in the skin}

Members of the eotaxin subfamilies are well-known to have a major chemotactic role for eosinophils. Recent advances in this field are the significant correlation shown between the level of the chemokine (C-C motif) ligand 24 (CCL24)/eotaxin 2 and the degree of eosinophilia and clinical symptoms in patients with rhinitis or with allergy [9]. Expression of 
CCL24 in the nasal mucosa of allergic and asthmatic patients was significantly increased as compared to control and COPD groups [9]. Furthermore, the up-regulation of CCL26/eotaxin 3 was also enhanced in patients with seasonal allergic rhinitis leading to an increase of eosinophils in the nasal mucosa and probably in the airway walls of the lower respiratory tract [9]. Since skin eosinophil infiltration is one of the most important characteristics of atopic dermatitis, Bao et al. studied in detail the signal transduction pathway for IL-4 regulation of CCL26 in human immortalized keratinocytes [10]. They showed that the regulation is at the transcriptional level and involves the Janus kinase(jak)1 and Jak2/signal transducer and activator of transcription (Stat)6 pathway.

\section{Adhesion, migration and proliferation of eosinophils in the mucosa}

Recent papers focused on galectin-3 (Gal-3) and integrins. Gal-3, a $\beta$ galactoside binding lectin, is implicated in the pathogenesis of allergic airway inflammation. Gal-3 -/- mice exposed to chronic allergen (OVA) challenge have attenuated airway eosinophilia and exhibit less severe remodeling of the airways [11]. Furthermore, airway eosinophilia in acute and chronic allergen-challenged mice was accompanied with elevated levels of extracellular soluble Gal-3 in the BALF and intracellular Gal-3 in the lung tissue [11]. Gal-3 is secreted by various cell types such as macrophages and activated Th2 cells, but also by eosinophils especially when exposed to eotaxin-1. Secreted Gal-3 can bind to integrin and other glycan ligand on all the surface of human eosinophils to facilitate all trafficking and activation in allergic airway.

Arrest of eosinophils in vessels and their extravasation into the airway wall are partially mediated by the integrin family of cell adhesion receptors. Recent papers highlight for the first time the influence of different conformations of integrins on eosinophils trafficking. Studies in mice and human indicate that $\alpha_{4} \beta_{1}$ and $\alpha_{M} \beta_{2}$ are the major eosinophil integrins mediating cell adhesion with $\alpha_{4} \beta_{1}$ largely responsible for arrest of blood eosinophils on 
vascular cell adhesion molecule-1 (VCAM-1) in vessels of the asthmatic lung whereas activated $\alpha_{M} \beta_{2}$ by interacting with periostin and possibly other ligands, is involved in subsequent eosinophil recruitment and persistence in the extracellular matrix of the bronchi of asthma [12-14]. Cellular feature of eosinophils

New insights into immunity and Th2 immune responses were provided through a better understanding of the physiological roles of IL-5, which is produced by eosinophils and involved in many aspects of eosinophil development. Ikutani et al. [15] used a generation of IL-5/Venus knockin mice to identify innate IL-5-producing lymphoid cells that play a role in orchestrating eosinophil responses in the lung. They found unique tissue localization of innate IL-5-producing cells in the lungs of untreated mice; these cells regulate eosinophil infiltration into the lung, and may contribute to antitumor immunity via lung eosinophils.

IL-17 is an important cytokine of the T helper 17 pathway. It has been suggested that in the lung, IL-17 could be involved in the neutrophilic inflammation and airway remodeling of chronic respiratory diseases [16]. Eosinophils can promote IL-17 production through the release of IL-1 $\beta$ and modulates the allergic airway inflammation in asthma [17].

\section{NEW INSIGHTS IN THE BIOLOGY OF BASOPHILS}

Recent findings in the biology of basophils focus on their role in the regulation of $\mathrm{Th} 2$ cell response through IL-4 expression, in the differentiation of monocytes to macrophages, and on their population heterogeneity in human.

\section{Basophils and Th2 cell responses}

The apparent contradictions between different studies on the possibilities of basophils to act as APCs probably come from the nature and type of antigen presented and the route of antigens administration. Basophils alone were reported to polarize $\mathrm{T}$ cells towards $\mathrm{Th} 2$ for haptens and peptides antigens but needed the presence of DCs for protein antigens $[18,19]$. 
However, a basophil requirement for IL-4 production by naïve CD4+ T cell was not observed in novel reporter strains of mice following a papain-induced model of allergy [20]. In a very recent study, correlation between the numbers of basophils in the lungs tissues with IgEassociated airway allergic inflammation was reported in OVA-immunized mice, and purified pulmonary basophils from these mice were functional and amplified Th2 responses [21]. Furthermore, in vitro, lung basophils failed to initiate Ag-specific naïve or OVA-immunized but were able to cooperate with DCs to increase Ag-specific Th2 cell differentiation and to increase local Th2 response via IL-4-dependent and IL-4 independent mechanisms. Overall, both in vivo and in vitro, in humans as in mice, human basophils seem to enhance IL-4 expression by interacting directly with $\mathrm{CRTH} 2+\mathrm{CD} 4$ effector, circulating memory Th2 cells..

On the other hand, an unexpected interaction between basophils and monocytes-macrophages was observed during allergic skin inflammation [22]. The fate, polarization and function of monocytes after their recruitment to skin lesions were analyzed under an immunoglobulin Emediated chronic allergic inflammation (IgE-CAI), a model where basophils play a critical role. Basophils promoted the differentiation of inflammatory monocytes into M2 (alternatively activated) macrophages rather than M1 (classically activated) macrophages, being from proinflammatory to anti-inflammatory. By using new genetic tools, inflammatory monocytes were showed to acquire an M2-like phenotype through IL-4R in response to basophil-derived IL-4, and to exert an anti-inflammatory function to regulate the allergic inflammation. Furthermore, TSLP produced by basophils amplified the M2 polarization in allergic airway inflammation [23]. These findings bring new light on the role of basophils as regulators of allergic skin inflammation.

\section{Heterogeneity in basophils}

Whereas IL-3 is considered as essential for basophil activation, expansion and survival TSLP was recently reported to promote peripheral basophilia in both IL-3/IL-3-R- sufficient and 
deficient environments, and to contribute to Th2 cytokine responses in vivo (see Figure 1) [24]. Furthermore, genome-wide transcriptional profiling and functional analyses identified phenotypic heterogeneity, distinct responsiveness and functional potential between TSLPversus IL-3-elicited basophils. This heterogeneity in human basophil populations correlated with susceptibility to allergic inflammation [24].

\section{EOSINOPHIL AND BASOPHIL: WHAT DO THEY SHARE?}

Eosinophil development is determined by the interplay of a few transcription factors including PU.1, a member of the Ets (E-twenty six) transcription family, one of the largest families of transcription factors which have a conserved Ets domain that belongs to the winged helixturn-helix DNA-binding domain superfamily. PU.1 is involved in controlling transcription of eosinophil-specific genes such as $M P B$ (major basic protein), EDN (ribonuclease, RNase A family, 2), ECP (ribonuclease, RNase A family, 3), EPO (erythropoietin), CCR3 (chemokine (C-C motif) receptor 3) and $I L-5 R$ (Interleukin 5 receptor, alpha) [25]. PU.1 seems to have the same role in basophils since it was also involved in the transcriptional activity of the ST2 promoter - the Interleukin 1 receptor-like 1 gene (IL1RL1/ST2) encodes IL-33 receptor bound to its promoter region, and participated to its expression in human basophilic cell line $[26]$.

Finally, because little is known about the mechanism of cooperative function between basophils and eosinophils in immunoglobulin E-mediated chronic allergic inflammation (IgECAI), Matsuoka et al. [27] generated new basophil- and eosinophil-depletion mouse models (basophil diphtheria toxin receptor (basoDTR) and eosinophil (Eo)DTR mice respectively). As said by the authors, the basoDTR mice model will permit to measure the cellular dynamics of basophils, and the EoDTR mice is the first model for conditional eosinophil ablation in vivo. 
The recent genetic findings at both the candidate and the genome-wide levels are now discussed. The main new results in candidate genetic studies are the genes involved in the intracellular calcium influx and apoptosis in eosinophils. At the genome-wide level, studies identified genetic variants related to the triade of cytokines that we described in the first subsection.

\section{Candidate association studies}

Eosinophils and basophils are known to be heritable traits with heritability estimate ranged from $14 \%$ for basophils to $30-35 \%$ for eosinophil counts [28,29]. To our knowledge, no association between genes and basophil counts or percent was investigated at a candidate level. Recent studies on eosinophils have explored genes in two pathways involved in the cellular response which are the intracellular calcium influx and apoptosis. Following the establishment of the importance of the 17q12-21 locus in asthma, which includes the gasdermin B (GSDMB)/ORM1-like 3 (ORMDL3) genes, Kang et al. [30] investigated the association between polymorphisms in these genes, whose ORMDL3 protein binds to and inhibits the calcium pump in the sarco-endoplasmic reticulum, and blood eosinophils expressed as percent in 1907 elementary school Korean children. Eosinophils were significantly lower in subjects carrying the A allele of rs4794820 and with a methacholine provocation test value $\leq 16$, showing for the first time that polymorphisms in the GSDMB/ORMDL3 genes may affect eosinophil-mediated bronchial hyperresponsiveness. Another interesting but intriguing result is the association of the rs2238634T/rs2238633G/rs2238632T/rs2238631A haplotype in the thromboxane A2 receptor gene $(T B X A 2 R)$, member of the seven-transmembrane G-protein-coupled receptor super family, and blood eosinophils in 226 childhood-onset asthma patients and 164 controls from Japan: homozygotes for this haplotype had higher levels of blood eosinophils than other zygotes in female controls, whereas a trend for lower levels of blood eosinophils was found in 
female asthma patients. No association was reported in male whatever the asthma status [31]. Furthermore, this haplotype was associated with lower lung function in asthma patients, and with increased $T B X A 2 R$ mRNA level, suggesting severe airway inflammation and/or remodeling in these subjects. The last study [32] investigated association between Baculoviral IAP repeat containing 5 (BIRC5, also called survivin), a member of the inhibitor of apoptosis protein family and blood eosinophils in a case-control study (307 asthma /344 control Hungarian subjects). The authors found that individuals homozygous for the wild-type allele of rs9904341 had elevated eosinophil levels compared to those carrying either one or two copy of the rare allele. This result was confirmed by haplotype analysis, and was coherent with the elevated BIRC5 mRNA levels found in sputum samples of patients with asthma as compared to controls, and the correlation between sputum eosinophil and BIRC5 mRNA levels, suggesting that survivin may have a primordial role in eosinophil accumulation. Overall, these finding highlight the interest to study several genes in metabolic pathway such as regulation of calcium influx and apoptotic response to better understand the contribution of eosinophils in allergic inflammation.

\section{Genome-wide association studies}

Three genome-wide association studies (GWAS) of eosinophil and/or basophil cell counts have been conducted in European and Asian populations [33-35]. These GWAS led to detection of four loci with pleiotropic effect on eosinophil and basophil counts in both European and Asian samples: GATA2 (GATA binding protein 2, 3q21), MHC region (major histocompatibility complex, 6p21), HBS1L-MYB (HBS1-like - myeloblastosis viral oncogene homolog, 6q23), and ERG (erythroblastosis virus E26 oncogene homolog, 21q22) [33,35]. Three other regions showed association with basophil count: SLC45A3-NUCKS1 (solute carrier family 45 , member 3 - nuclear casein kinase and cyclin-dependent kinase substrate 1 , 1q32), RPN1-C3orf27 (ribophorin I - chromosome 3 open reading frame 27, 3p21), and 
NAALAD2 (N-acetylated alpha-linked acidic dipeptidase 2, 11q14) [34,35]. The first GWAS of eosinophils identified six other regions associated with eosinophil counts in European populations: IL1RL1 (2q12), IKZF2 (IKAROS family zinc finger 2, 2q13), WDR36 (WD repeat domain 36, 5q22), IL5 (interleukin 5, 5q31), IL33 (interleukin 33, 9p24), and SH2B3 (SH2B adaptor protein 3, 12q24) [33]. Interestingly, in this study, the genetic variants in IL1RL1, WDR36 and IL33, that were associated first with eosinophil count, were also associated with asthma and atopic asthma [33]. Moreover, a GWAS of eosinophilic esophagitis (a food allergy-related disorder characterized by the accumulation of eosinophils in the esophagus) identified genetic variants belonging to TSLP-WDR36 locus among the most significant SNPs associated with the disease [36]. Candidate gene studies of IL33, TSLP and ILIRL1 as well as GWAS of asthma and asthma-related phenotypes have further implicated these loci in asthma and atopy susceptibility.

\section{CONCLUSION}

In the last years, the immunological approach has considerably developed the understanding of the cellular mechanisms of asthma and allergy. One could ask if allergy is really an immunological disease? and asthma a genetic disorder affecting the bronchial smooth muscle? The eosinophils play a key role in allergic inflammation but other cells such as basophils are still poorly known to be involved in the pathway leading to the inflammatory reaction. A lot of extensive studies have been done in mice to better understand the underlying immunological mechanisms, but very few papers have investigated the human cells. Novel genetic tools are developed in mice models to elucidate the role of basophils in vivo. It is difficult to anticipate if the results will reflect some functional significances similar as those in humans. Furthermore, the large number of different clinical phenotypes observed in asthma and allergy complicates the understanding of the immunological mechanisms. 
The genetic studies highlight the importance of epithelial cell-derived cytokines (TSLP and IL-33) that promote differentiation and activation of Th2 cells and their receptors (IL1RL1 encodes ST2, the receptor for IL-33 on immune cells including mast cells, Th2 cells, T regulatory cells). Replication of genetic results is often difficult due to the disparity in phenotype definition. A pathway-based approach where several genes are selected for analysis, based on biological knowledge, can represent a good strategy to detect relevant associations with eosinophils or basophils, and better understand their role in allergic inflammation. 


\section{Key bullet points}

Key bullet point 1: New epithelial cell-derived cytokines, including IL-25, IL-33 and TSLP activate innate immune cells such as basophils and eosinophils.

Key bullet point 2: Galectin-3 and different conformations of integrins regulate trafficking and activation of eosinophils in allergic airways.

Key bullet point 3: Acting as antigen-presenting cells for haptens and peptide antigens, basophils interact with Th2, monocytes and macrophages, thus taking an ever increasingly importance in the regulation of the allergic skin inflammation.

Key bullet point 4: Biological and genetic studies highlight the role of epithelial cell-derived cytokines (TSLP and IL-33) that promote differentiation and activation of th2 cells and their receptors.

\section{Please add acknowledgements if applicable}

Research projects in UMRS 1018 \& EA4483 are supported by grants from the French Research Agency (ANR CES-2009), and program of the "Nord pas de Calais". RM \& FZ received financial supports from the University Lille 2.

\section{Please add conflicts of interest; if there are none, please state so}

There are no conflicts of interest for any of the authors in relation to this publication.

Figure. 1. Cascade of events involving eosinophils and basophils related to immunological responses in allergy. The cellular mechanisms of asthma and allergy are complex in the bronchial mucosa. Many mediators are released inducing smooth muscle contraction and local inflammation. Epithelial cell activation by inhaled allergen activates the local network of 
dendritic cells which promote Th2 immune responses. Among the cytokines released by epithelial cells, IL-25, IL-33 and TSLP seem to play an important role in in the pathway leading to the inflammatory reaction. For example, TSLP promotes the growth and differentiation of basophils from the bone marrow whereas IL-25 and IL-33 enhance Th2 development that mainly produce IL-5 and IL-13 and contribute to eosinophilic inflammation. 


\section{REFERENCES AND RECOMMENDED READING}

Papers of particular interest, published within the annual period of review, have been highlighted as:

* of special interest

** of outstanding interest

Additional references related to this topic can also be found in the Current World Literature section in this issue (pp. $\mathrm{xx}-\mathrm{xx})$.

1. Falcone FH, Haas H, Gibbs BF. The human basophil: a new appreciation of its role in immune responses. Blood 2000; 96:4028-4038.

2. Fulkerson PC, Rothenberg ME. Targeting eosinophils in allergy, inflammation and beyond. Nat Rev Drug Discov 2013; 12:117-129.

3*. Lambrecht BN, Hammad H. The airway epithelium in asthma. Nat Med 2012; 18:684692.

The review clearly shows that the airway epithelium controls many aspects of allergic sensitization and play an important role in allergic inflammation, remodeling and hyperreactivity. Thus, the epithelial cells are involved in the immunological pathways.

4*. Bartemes KR, Kita H. Dynamic role of epithelium-derived cytokines in asthma. Clin Immunol 2012; 143:222-235.

The airway epithelium play an important role in driving Th2 type immune response and chronic airway inflammation. New epithelial cell-derived cytokines, including IL-25, IL-33 and TSLP are implicated in Th2-type immune responses.

5. Corrigan CJ, Wang W, Meng Q, et al. Allergen-induced expression of IL-25 and IL-25 receptor in atopic asthmatic airways and late-phase cutaneous responses. J Allergy Clin Immunol 2011; 128:116-124. 
6. Haenuki Y, Matsushita K, Futatsugi-Yumikura S, et al. A critical role of IL-33 in experimental allergic rhinitis. J Allergy Clin Immunol 2012; 130:184-194 e111.

7. Cho KA, Suh JW, Sohn JH, et al. IL-33 induces Th17-mediated airway inflammation via mast cells in ovalbumin-challenged mice. Am J Physiol Lung Cell Mol Physiol 2011; 302:L429-440.

8. Headley MB, Zhou B, Shih WX, et al. TSLP conditions the lung immune environment for the generation of pathogenic innate and antigen-specific adaptive immune responses. J Immunol 2009; 182:1641-1647.

9. Paplinska M, Hermanowicz-Salamon J, Nejman-Gryz P, et al. Expression of eotaxins in the material from nasal brushing in asthma, allergic rhinitis and COPD patients. Cytokine $2012 ; 60: 393-399$.

10. Bao L, Shi VY, Chan LS. IL-4 regulates chemokine CCL26 in keratinocytes through the Jak1, 2/Stat6 signal transduction pathway: Implication for atopic dermatitis. Mol Immunol 2012; 50:91-97.

11. Ge XN, Ha SG, Liu FT, et al. Eosinophil-expressed galectin-3 regulates cell trafficking and migration. Front Pharmacol 2013; 4:37.

12. Johansson MW, Gunderson KA, Kelly EA, et al. Anti-IL-5 attenuates activation and surface density of beta(2) -integrins on circulating eosinophils after segmental antigen challenge. Clin Exp Allergy 2013; 43:292-303.

13*. Johansson MW, Mosher DF. Integrin activation States and eosinophil recruitment in asthma. Front Pharmacol 2013; 4:33

This is an important work that demonstrates that activation states of eosinophil $\beta 1$ and $\beta 2$ integrins correlate with measurements of eosinophil recruitment and pulmonary function in asthma. 
14. Masuoka M, Shiraishi H, Ohta S, et al. Periostin promotes chronic allergic inflammation in response to Th2 cytokines. J Clin Invest 2012; 122:2590-2600.

15. Ikutani M, Yanagibashi T, Ogasawara M, et al. Identification of innate IL-5-producing cells and their role in lung eosinophil regulation and antitumor immunity. $\mathrm{J}$ Immunol $2012 ; 188: 703-713$.

16. Tan HL, Rosenthal M. IL-17 in lung disease: friend or foe? Thorax 2013.

17. Esnault S, Kelly EA, Nettenstrom LM, et al. Human eosinophils release IL-1ss and increase expression of IL-17A in activated CD4+ T lymphocytes. Clin Exp Allergy $2012 ; 42: 1756-1764$.

18. Eckl-Dorna J, Ellinger A, Blatt K, et al. Basophils are not the key antigen-presenting cells in allergic patients. Allergy 2012; 67:601-608.

19*. Otsuka A, Nakajima S, Kubo M, et al. Basophils are required for the induction of Th2 immunity to haptens and peptide antigens. Nat Commun 2013; 4:1738.

This is an important work that demonstrates that basophils alone can induce Th2-type immune responses for exposure to haptens or peptide antigens but must cooperate with PC cells for the induction Th2 for exposure to protein allergens.

20. Sullivan BM, Liang HE, Bando JK, et al. Genetic analysis of basophil function in vivo. Nat Immunol 2011; 12:527-535.

21. Wakahara K, Van VQ, Baba N, et al. Basophils are recruited to inflamed lungs and exacerbate memory Th2 responses in mice and humans. Allergy 2013; 68:180-189.

22**. Egawa M, Mukai K, Yoshikawa S, et al. Inflammatory monocytes recruited to allergic skin acquire an anti-inflammatory M2 phenotype via basophil-derived interleukin-4. Immunity 2013; 38:570-580.

This study reports how basophils drive the differentiation of inflammatory monocytes into M2 (alternatively activated) macrophages thereby regulating allergic skin inflammation. 
23. Han H, Headley MB, Xu W, et al. Thymic stromal lymphopoietin amplifies the differentiation of alternatively activated macrophages. J Immunol 2013; 190:904-912.

24. Siracusa MC, Saenz SA, Hill DA, et al. TSLP promotes interleukin-3-independent basophil haematopoiesis and type 2 inflammation. RNA 2012; 477:229-233.

25. Uhm TG, Kim BS, Chung IY. Eosinophil development, regulation of eosinophil-specific genes, and role of eosinophils in the pathogenesis of asthma. Allergy Asthma Immunol Res 2012; 4:68-79.

26*. Baba Y, Maeda K, Yashiro T, et al. Involvement of PU.1 in mast cell/basophil-specific function of the human IL1RL1/ST2 promoter. Allergol Int 2012; 61:461-467.

The transcription factor PU.1 binds to the IL1R1/ST2 promoter and participates in its expression.

27*. Matsuoka K, Shitara H, Taya C, et al. Novel basophil- or eosinophil-depleted mouse models for functional analyses of allergic inflammation. PLoS One 2013; 8:e60958.

The two mouse models generated in this study clearly distinguish the in vivo functions of basophils and eosinophils in IgE-chronic Allergic Inflammation, providing a new approach for clarifying the role of these cells in allergic inflammation.

28. Bouzigon E, Chaudru V, Carpentier AS, et al. Familial correlations and inter-relationships of four asthma-associated quantitative phenotypes in 320 French EGEA families ascertained through asthmatic probands. Eur J Hum Genet 2004; 12:955-963.

29. Pilia G, Chen WM, Scuteri A, et al. Heritability of cardiovascular and personality traits in 6,148 Sardinians. PLoS Genet 2006; 2:e132.

30*. Kang M-J, Yu H-S, Seo J-H, et al. GSDMB/ORMDL3 variants contribute to asthma susceptibility and eosinophil-mediated bronchial hyperresponsiveness. Human Immunology 2012; 73:954-959. 
This study replicates previous findings that identified a relationship between the GSDMB/ORMDL3-bearing locus, 17q12-21 and asthma, and suggests for the first time that GSDMB/ORMDL3 polymorphisms may shape the development of eosinophil-mediated bronchial hyperresponsiveness in Korean children from the general population.

31. Takeuchi K, Mashimo Y, Shimojo N, et al. Functional variants in the thromboxane A2 receptor gene are associated with lung function in childhood-onset asthma. Clin Exp Allergy 2013; 43:413-424.

32. Ungvari I, Hadadi E, Virag V, et al. Implication of BIRC5 in asthma pathogenesis. Int Immunol 2012; 24:293-301.

33. Gudbjartsson DF, Bjornsdottir US, Halapi E, et al. Sequence variants affecting eosinophil numbers associate with asthma and myocardial infarction. Nat Genet 2009; 41:342347.

34. Nalls MA, Couper DJ, Tanaka T, et al. Multiple loci are associated with white blood cell phenotypes. PLoS Genet 2011; 7:e1002113.

35. Okada Y, Hirota T, Kamatani Y, et al. Identification of nine novel loci associated with white blood cell subtypes in a Japanese population. PLoS Genet 2011; 7:e1002067.

36. Rothenberg ME, Spergel JM, Sherrill JD, et al. Common variants at 5q22 associate with pediatric eosinophilic esophagitis. Nat Genet 2010; 42:289-291. 


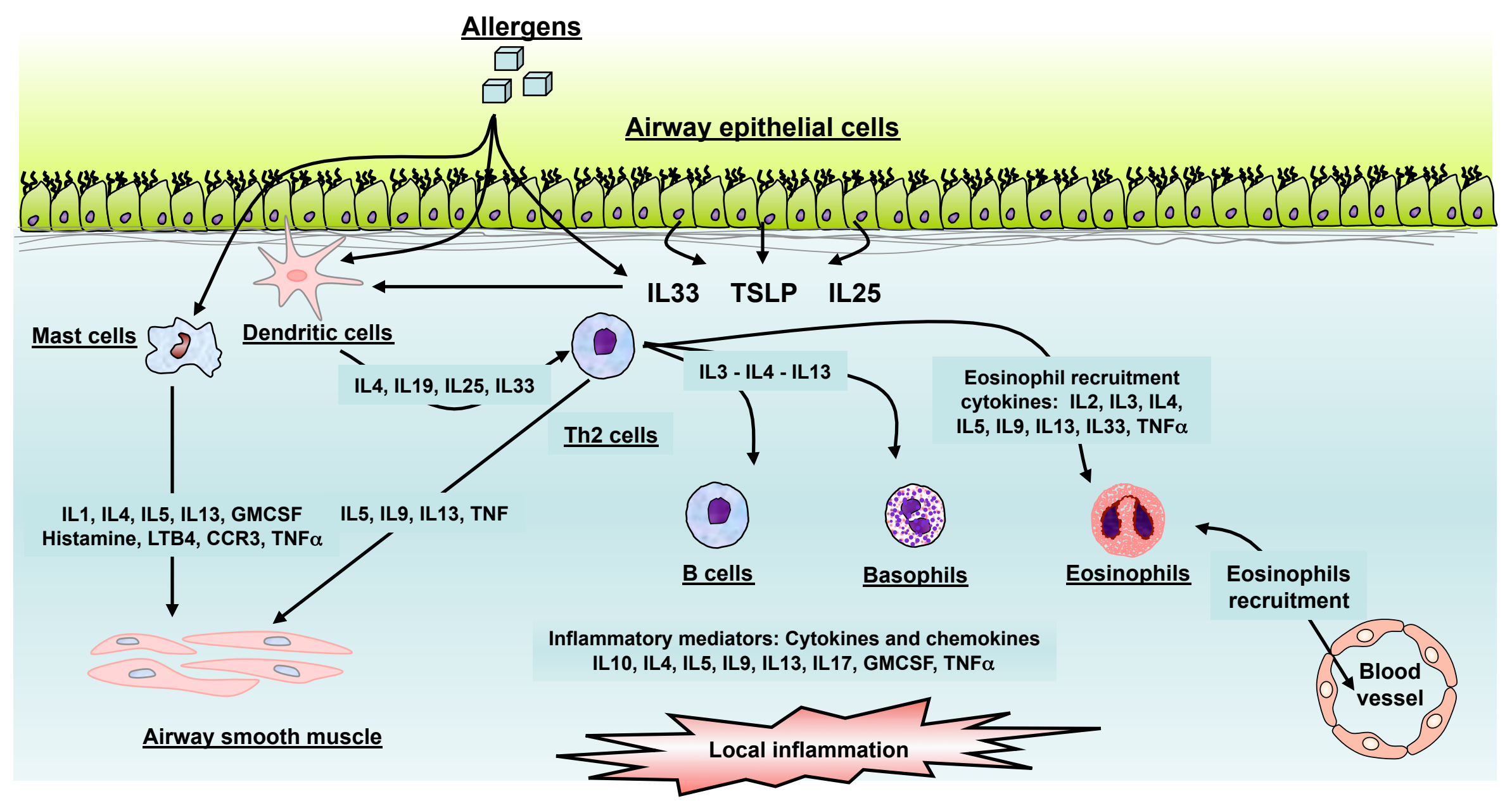

